INNOVATIONS IN PRIMARY CARE

\title{
Huddling Up: Expanding Clinic Huddles
}

Ann Tseng, MD

Ann Fam Med 2017;15:584. https://doi.org/10.1370/afm.2156

\section{THE INNOVATION}

Huddles are a common tool in primary care practices that support team-based care. ${ }^{1,2}$ We describe our expansion of huddles beyond the standard medical assistant/clinician huddles in 4 ways: front desk/clinician; behavioral health/clinician; registered nurse care manager/clinician; and clinic leadership.

\section{WHO AND WHERE}

We have utilized medical assistant/clinician huddles before the start of a clinic session for many years now. During this brief huddle, the teamlet anticipates and plans for needs during the patient session. In a large practice of over 80 employees, however, we realize the need for better communication with all our team members beyond the medial assistant/clinician teamlet.

Through both a literature review and a multidisciplinary committee of composed of representatives from the front desk, registered nurse, behavioral health, and clinic leadership groups, we created the structure of our expanded huddles. Front desk/clinician huddles happen in either in the clinician's office or at the front desk employee's workstation. Behavioral health/clinician huddles and registered nurse/clinician huddles happen in our main clinical care area during all our patient care sessions. Finally, clinic leadership huddles happen in our administrative office hallway, in front of a visual board that houses operational information.

Conflicts of interest: autbor reports none.

\section{CORRESPONDING AUTHOR}

Ann Tseng, MD

Oregon Health and Science University

Family Medicine at Gabriel Park

4411 SW Vermont Ave

Portland OR, 97219

tsenga@ohsu.edu

\section{HOW}

Front desk/clinician huddles happen weekly for around 10 to 15 minutes. A specific agenda is followed. The huddle starts with appreciation where each person expresses appreciation for each other formally. Any goals are then discussed to improve care. Finally, the clinician schedule is reviewed for the upcoming week for any changes needed in scheduling to optimize both the patient experience and the clinician's workflow.

Behavioral health/clinician huddles happen daily and last approximately 5 minutes with each clinician, before or during the clinic session. The goal is to better plan for anticipated behavioral health needs for patients with scheduled appointments.

Registered nurse care manager/clinician huddles happen daily and last approximately 5 minutes with each clinician. They happen before or during the clinic session to better anticipate care coordination and care management needs for scheduled patients.

Clinic leadership huddles happen daily and last approximately 15 minutes. The practice manager, medical director, leads for front and back office, registered nurse, and behavioral health team meet to discuss clinical operations. The goals are to review and plan for adequate open appointments, unexpected absences of staff and providers, and urgent operational concerns. Plans for the next day's operations are also reviewed.

\section{LEARNING}

Huddles have improved communication, teamwork, and efficiency between staff and clinicians in our practice. They also improve engagement with both the patient and the clinic. Finally, huddles have built a culture of appreciation amongst our staff and clinicians. We have found a brief, scheduled huddle with a clear agenda maximizes the effectiveness of this intervention in our practice. ${ }^{1,2}$

Author affiliations, references, and the supplemental materials are available at http://www.annfammed.org/content/15/6/584I suppl/DC1. 